\title{
Intravenous Milrinone Salvage Therapy in Acute Internal Carotid Artery Occlusion: Case Report
}

Hosam Al-Jehani (D) - Mark Angle

Received: October 20, 2018 / Published online: January 19, 2019

(C) The Author(s) 2019

\begin{abstract}
Introduction: Acute internal carotid artery occlusion is a devastating form of ischemic accident with significant morbidity and possible mortality. The "threatened hemisphere" is allowed limited access to collateral circulation further worsening the ischemic burden and the overall prognosis.

Methods: We present the details of a case of a 38-year-old woman who suffered from an ischemic stroke. The clinical course of which showed a hemodynamic dependence to preserve her neurological function, prompting the use of the phosphodiesterase inhibitor milrinone to maintain her neurological function.

Result: This case represents the first case in the literature in which a medical therapy is used to salvage brain tissue in the site of acute symptomatic large vessel occlusion. This has spared
\end{abstract}

Enhanced Digital Features To view enhanced digital features for this article go to https://doi.org/10.6084/ m9.figshare.7479875.

H. Al-Jehani $(\bowtie) \cdot$ M. Angle

Neurocritical Care Unit, Montreal Neurological Institute/Hospital, McGill University, Montreal, Canada

e-mail: hjehani@iau.edu.sa

\section{H. Al-Jehani}

Department of Neurosurgery, King Fahad University Hospital, Imam Abdulrahman Bin Faisal University, Al-Khobar, Saudi Arabia the patient from a potential significant morbidity and even mortality if a revascularization procedure had been attempted.

Conclusion: This innovative use of milrinone, if applied in more captured patients with internal carotid occlusion or those with reversible hemodynamic failure, would improve our understanding of the ischemic thresholds and cerebral vascular reserves and would improve the clinical outcome of this significant ischemic insult.

Keywords: Ischemic cerebrovascular accident; Internal carotid artery occlusion; Milrinone; Phosphodiesterase inhibitor

\section{INTRODUCTION}

Acute internal carotid artery occlusion is a devastating neurological emergency accounting for about $10 \%$ of all ischemic cerebrovascular accidents [1]. Regardless of its etiology, including cardioembolic, atherothrombotic, or secondary to vessel-wall pathology, it is associated with a high burden of morbidity and mortality. Of those patients presenting with acute internal carotid artery occlusion, $16-55 \%$ will die, 40-60\% will suffer severe neurologic disability, and only $2-12 \%$ will achieve good functional recovery $[2,3]$. This bleak outcome is not offset by the utilization of intravenous rt-PA, since 
only $8 \%$ would achieve recanalization, altering the natural history of this entity [4].

Literature addressing the aggressive alternatives aimed at revascularization of the acutely symptomatic internal carotid artery is plagued by several controversial issues $[5,6]$. Technically feasible surgical intervention in the form of emergency carotid endarterectomy with distal thrombectomy has an elusive benefit and is not widely accepted $[7,8]$.

Although endovascular angioplasty with or without stent placement may be necessary to decrease underlying chronic vessel stenosis, it is not advocated in the site of acute occlusion for the higher complication rate and questionable benefit, since, despite a complete recanalization achieved in $64 \%$ of the cases, there still remains a 50\% mortality rate [9-11].

The overall approach to this subset of patients with what is considered a "threatened hemisphere" weighs heavily on the detrimental risk-benefit ratio with the fear of adding more insult to the injury in the event of successful revascularization.

Despite complete internal carotid occlusion, the affected hemisphere will recruit the precious blood supply by collateral flow through the various compartments of the circle of Willis [12]. The inter-individual variation in initiation and factors influencing the maintenance of these collateral flow channels in such situations results in profound variability in the neurological insult burden translated into clinical deficits due to circulatory hemodynamic failure or the so-called misery perfusion [13].

In such extreme conditions, the principles of cerebral autoregulation give rise to locoregional hyperemia allowing the recruitment of the collateral circulation to the affected brain region(s). These mechanisms are not fail-proof and the patients can deteriorate due to the failure of such an attempt $[13,14]$.

We recently published the $\mathrm{MNH}$ protocol, in which we employed the vasodilator effect of the phosphodiesterase inhibitor milrinone in cases of delayed ischemic neurological deficit with subarachnoid hemorrhage [15]. The vasodilator property of milrinone is proposed as a mechanism for promoting cortical-to-cortical shunting of cerebral blood flow to areas at risk, with significant reduction of cortical hypodensities and improved outcome in our cohort.

We present a case of young female who presented with an acute left carotid occlusion with profound subcortical neurological deficit, in whom milrinone infusion was used as a salvage therapy, achieving significant improvement in her global hemispheric blood flow with minimal residual deficits. This was carried out in full compliance with ethics guidelines and at no harm to the patient, and with no breach of confidentiality. Additional informed consent was obtained from all individual participants for whom identifying information is included in this article.

\section{CASE PRESENTATION}

A 38-year-old right-handed woman, originally from Sri Lanka, who was G2, P2, and A0 presented to our emergency department 2 weeks post-spontaneous vaginal delivery with a history of sudden onset of right arm jerking movement for 2 days lasting 2-3 min each. CT scans with and without contrast were carried out and showed no abnormalities, including no thrombus in the sagittal sinus. The patient had no events during a 12-h observation period in the emergency department and was sent home the next morning.

Five days later, the patient returned to the emergency department with sudden onset of right hemiplegia of the face, arm and leg with onset at 9:30 a.m. The hemiparesis began while she was standing up in the kitchen. She did not wake up with any symptoms, and she had no headache or seizure activity. There was no history of clotting disorder and no history of abortions, and there was no history of photosensitivity or rashes. She had had two spontaneous vaginal deliveries with no complications and both delivered at term. There was no history of alcohol or drug abuse. The patient is non-smoker and is not on any medications.

The clinical suspicion was high for cerebral sinus venous thrombosis. MRA and MRV did not reveal venous thrombosis. No parenchymal abnormality was noted on the MRI. The patient was admitted to the NICU for observation and 
was started on IV heparin, as the suspicion was that this patient did have a small thrombus that was overlooked by imaging.

The patient was alert and oriented with a stable hemodynamic and respiratory status. She had a dysarthric speech with intact comprehension and expression. Pupils were equal and reactive to light and accommodation. There was no INO and no relative afferent pupillary defect. There was no nystagmus. EOMs were normal. Visual fields were normal to confrontation. There was decreased sensation to light touch and pinprick in the right V2 and V3 distribution. There was a right upper motor neuron facial weakness. Cranial nerves 9, 10, and 11 were normal. The tongue was deviated to the right.

Motor examination revealed decreased tone on the right upper and lower extremities. Bulk was normal. Fine-finger movement was not present in the right hand. There was upper and lower extremity weakness in the corticospinal distribution at the level of $1-2 / 5$. Reflexes were diffusely brisk, but more so on the right with positive Hoffman's sign bilaterally, with a clear right up-going toe.

Sensory examination revealed decreased sensation in all modalities to the right side. Coordination examination was limited by the weakness on the right side but was normal on the left. It was not possible to examine gait.

This clinical examination localized the lesion to a left subcortical event, either in the corona radiata or the internal capsule. Review of the MRI revealed some ischemic changes in this region and a totally occluded left internal carotid artery. For that, and her overall clinical condition, IV t-PA was deferred.

Further MRA analysis revealed the site of the occlusion of the left internal carotid artery to be just above the bifurcation, and this most likely represented carotid dissection. Doppler ultrasound was carried out confirming this finding and also revealed significant vascular disease in both vertebral arteries. A digital subtraction cerebral angiogram was carried out that confirmed severe 3-vessel disease with fibromuscular dysplasia affecting both vertebral arteries and the right internal carotid artery, with a completely occluded left internal carotid artery with no run-off flow seen in the left middle cerebral artery.

A SPECT study was carried out showing symmetrical perfusion in both hemispheres, a finding which, coupled with the relatively stable course, deferred the decision for urgent surgical or interventional revascularization (Fig. 1).

The patient continued on the IV heparin. She interestingly developed a pattern of recurrent worsening right hemiplegia with no hemodynamic explanation (BP 150/70) and despite being appropriately anticoagulated (PTT $>80)$. In an attempt to preserve and improve global cerebral blood flow, we instituted IV milrinone therapy as per our NICU protocol ( $5 \mathrm{mg}$ bolus followed by $0.75 \mu \mathrm{g} / \mathrm{kg} /$ min infusion). This resulted in significant improvement in her right-sided power such that the next morning the patient had a near-normal power in the right side with a slight pronator drift and was able to ambulate. The patient was maintained on the milrinone and heparin drips. After 7 days of infusion, the milrinone was weaned with significant recurrence of her hemiplegic symptoms with significant weakness in the right side, necessitating a new bolus of milrinone of $3 \mathrm{mg}$ with a return of the basal rate of milrinone of $0.75 \mu \mathrm{g} / \mathrm{kg} / \mathrm{min}$

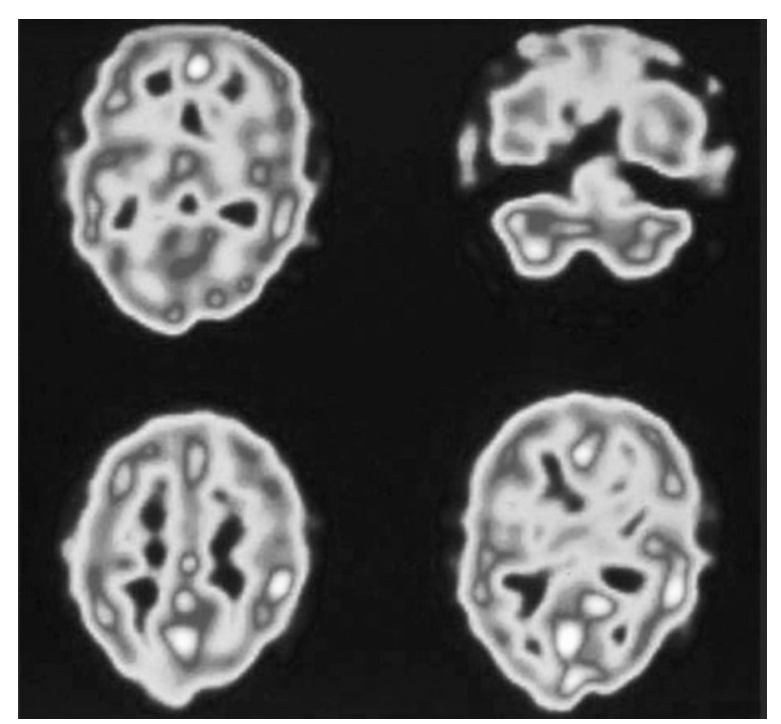

Fig. 1 SPECT study showing symmetrical hemispheric perfusion despite the left internal carotid occlusion 
with resolution of her deficits. After a total of 14 days of milrinone infusion, a SPECT scan was repeated revealing preserved global hemispheric blood flow. On the 15th day, milrinone was weaned off with no recurrence of her weakness. She was maintained on the heparin drip and observed for $36 \mathrm{~h}$ off milrinone with no recurrence of her symptoms. A SPECT scan was repeated at this time showing normal perfusion of both hemispheres.

The patient was transferred to the neurology ward and was switched to coumadin. She continued to improve in her fine motor skills for which she was discharged to an outpatient rehabilitation center. She continues to follow in our outpatient clinic showing no deficits and is currently maintained on $81 \mathrm{mg}$ of aspirin daily.

\section{DISCUSSION}

Acute symptomatic internal carotid artery occlusion is a neurological catastrophe. Despite available therapies, the outcome remains poor with high morbidity and mortality. It is an extreme condition that does not lend itself easily to aggressive surgical or endovascular therapies for fear of additional complications and concerns of the futility of intervention. Our patient posed an additional layer of difficulty being at such a young age with no precedent and with significant vessel-wall disease as the triggering factor, increasing the already high risk of any potential therapeutic intervention. Her stroke was stable initially, prompting an initial observatory approach. Then, she continued to deteriorate without evidence of hemodynamic deraignment, suggesting failure of her compensatory autoregulatory mechanism to maintain global left hemispheric cerebral blood flow. There was no hemodynamic or rheological factor to optimize since she had an appropriate mean arterial blood pressure and was fully anticoagulated. With these factors to consider, we employed the vasodilator effect of the phosphodiesterase inhibitor milrinone in attempt to ameliorate the autoregulation deraignment manifesting as progressive neurological deterioration. Interestingly, the patient responded to a near normal examination, suggesting that the significant majority of her deficit was attributed to cerebral hemodynamic failure or misery perfusion despite the presence of collateral flow. We hypothesize that the effect of the milrinone in this condition is that of cortical to cortical/subcortical blood flow augmentation to compensate for the failed autoregulation mechanisms in play in the areas at risk. The observations that the patient had a recurrence of her symptoms after 1 week of milrinone infusion, and became independent of milrinone after 15 days of infusion, are of great interest. We hypothesize that the milrinone bridged the period of autoregulation failure and helped re-establish the hemodynamic thresholds in the area at risk to better preserve and maintain function. This spared the patient the potential for significant morbidity and even mortality if a revascularization procedure had been attempted. This would be of great added value to stroke patients who are not attended to within the window of thrombolysis or thrombectomy, yet having enough collaterals to preserve their vulnerable brain tissue.

\section{CONCLUSION}

This case represents the first report in the literature in which a medical therapy is used to salvage brain tissue in the site of acute symptomatic large vessel occlusion. With more cases captured, through the combination of non-invasive vascular and hemodynamic autoregulatory imaging of the brain, this strategy could prove of great benefit to the select group of patients who are at risk of significant and irreversible neurological morbidity and mortality due to complete symptomatic large cerebral vessel occlusion.

\section{ACKNOWLEDGEMENTS}

Funding. No funding or sponsorship was received for this article or the article processing charges. The Article Processing Charges were funded by the authors. 
Disclosures. Hosam Al Jehani and Mark Angles have nothing to disclose.

Authorship. All named authors meet the International Committee of Medical Journal Editors (ICMJE) criteria for authorship for this article, take responsibility for the integrity of the work as a whole, and have given their approval for this version to be published.

Compliance with the Ethics Guidelines. Additional informed consent was obtained from all individual participants for whom identifying information is included in this article.

Open Access. This article is distributed under the terms of the Creative Commons Attribution-NonCommercial 4.0 International License (http://creativecommons.org/licenses/ by-nc/4.0/), which permits any noncommercial use, distribution, and reproduction in any medium, provided you give appropriate credit to the original author(s) and the source, provide a link to the Creative Commons license, and indicate if changes were made.

\section{REFERENCES}

1. Adams HP Jr, Bendixen BH, Leira E, et al. Antithrombotic treatment of ischemic stroke among patients with occlusion or severe stenosis of the internal carotid artery: a report of the Trial of Org 10172 in Acute Stroke Treatment (TOAST). Neurology. 1999;53:122-5.

2. Meyer FB, Sundt TM Jr, Piepgras DG, Sandok BA, Forbes G. Emergency carotid endarterectomy for patients with acute carotid occlusion and profound neurological deficits. Ann Surg. 1986;203:82-9.

3. Sugg RM, Malkoff MD, Noser EA, et al. Endovascular recanalization of internal carotid artery occlusion in acute ischemic stroke. Am J Neuroradiol. 2005;26:2591-4.

4. Tissue Plasminogen Activator for Acute Ischemic Stroke. The national institute of neurological disorders and stroke rt-PA stroke study group. N Engl J Med. 1995;333:1581-7.
5. Weis-Muller BT, Huber R, Spivak-Dats A, Turowski B, Siebler M, Sandmann W. Symptomatic acute occlusion of the internal carotid artery: reappraisal of urgent vascular reconstruction based on current stroke imaging. J Vasc Surg. 2008;47:752-9.

6. Li AH, Wang $\mathrm{YH}, \mathrm{Kao} \mathrm{HF}$, et al. Aggressive revascularization of acute internal carotid artery occlusion in patients with NIHSS $>20$ and poor collateral circulation: preliminary report. Int J Cardiol. 2011;161:97-102.

7. Kasper GC, Wladis AR, Lohr JM, et al. Carotid thromboendarterectomy for recent total occlusion of the internal carotid artery. J Vasc Surg. 2001;33:242-9.

8. Sbarigia E, Toni D, Speziale F, et al. Emergency and early carotid endarterectomy in patients with acute ischemic stroke selected with a predefined protocol. A prospective pilot study. Int Angiol. 2003;22:426-30.

9. Zaidat OO, Suarez JI, Santillan C, et al. Response to intra-arterial and combined intravenous and intraarterial thrombolytic therapy in patients with distal internal carotid artery occlusion. Stroke. 2002;33:1821-6.

10. CARESS Steering Committee. Carotid revascularization using endarterectomy or stenting systems (CARESS): phase I clinical trial. J Endovasc Ther. 2003;10:1021-30.

11. Yadav JS, Wholey MH, Kuntz RE, et al. Protected carotid-artery stenting versus endarterectomy in high-risk patients. $\mathrm{N}$ Engl J Med. 2004;351:1493-501.

12. Thanvi B, Robinson T. Complete occlusion of extracranial internal carotid artery: clinical features, pathophysiology, diagnosis and management. Postgrad Med J. 2007;83:95-9.

13. Yamauchi H, Higashi $T$, Kagawa $S$, et al. Is misery perfusion still a predictor of stroke in symptomatic major cerebral artery disease? Brain. 2012;135:2515-26.

14. Mangla R, Kolar B, Almast J, Ekholm SE. Border zone infarcts: pathophysiologic and imaging characteristics. Radiographics. 2011;31:1201-14.

15. Lannes M, Teitelbaum J, del Pilar Cortes M, Cardoso M, Angle M. Milrinone and homeostasis to treat cerebral vasospasm associated with subarachnoid hemorrhage: the Montreal Neurological Hospital protocol. Neurocrit Care. 2012;16:354-62. 